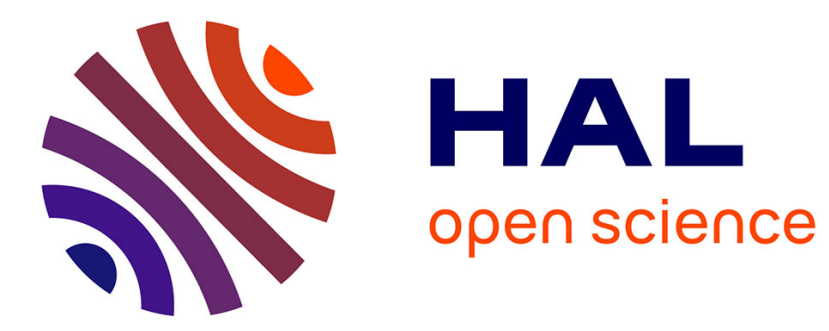

\title{
Homography-based Visual Servoing for Autonomous Underwater Vehicles
}

Minh-Duc Hua, Guillaume Allibert, Szymon Krupínski, Tarek Hamel

\section{To cite this version:}

Minh-Duc Hua, Guillaume Allibert, Szymon Krupínski, Tarek Hamel. Homography-based Visual Servoing for Autonomous Underwater Vehicles. Proceedings of the 19th IFAC World Congress, 2014, Aug 2014, Cape Town, South Africa. 10.3182/20140824-6-ZA-1003.01665 . hal-01075038

\section{HAL Id: hal-01075038 https://hal.science/hal-01075038}

Submitted on 16 Oct 2014

HAL is a multi-disciplinary open access archive for the deposit and dissemination of scientific research documents, whether they are published or not. The documents may come from teaching and research institutions in France or abroad, or from public or private research centers.
L'archive ouverte pluridisciplinaire HAL, est destinée au dépôt et à la diffusion de documents scientifiques de niveau recherche, publiés ou non, émanant des établissements d'enseignement et de recherche français ou étrangers, des laboratoires publics ou privés. 


\title{
Homography-based Visual Servoing for Autonomous Underwater Vehicles
}

\author{
Minh-Duc Hua* Guillaume Allibert** Szymon Krupínski*** \\ Tarek Hamel ${ }^{* *}$ \\ * Institut des Systèmes Intelligents et de Robotique (ISIR) \\ UPMC-CNRS, Paris, France (e-mail: hua@isir.upmc.fr) \\ ** I3S UNSA-CRNS, Sophia-Antipolis, France \\ (e-mails: allibert(thamel)@i3s.unice.fr) \\ *** Cybernetix, Marseille, France \\ (e-mail: szymon.krupinski@cybernetix.fr)
}

\begin{abstract}
A nonlinear visual servoing approach is proposed for the stabilisation of fullyactuated autonomous underwater vehicles (AUVs) by exploiting the homography matrix between the two images of a planar scene. In a cascade manner, an outer-loop control defines a reference setpoint based on the homography matrix, and an inner-loop control ensures the stabilisation of the setpoint by assigning thrust and torque controls. In contrast with conventional kinematic solution, the proposed controller deals with the high nonlinearity and coupling of the system dynamics and ensures almost-global asymptotical stability. In addition, the interactions of the AUV with the surrounding fluid (e.g., added mass and drag effects) are often difficult to model precisely whereas they may significantly perturb its motion. The proposed controller -augmented with an effective integral action- allows for the compensation of model uncertainties and for robust performance against such perturbations. Simulation results illustrating these properties on a realistic AUV model subject to sea current are reported.
\end{abstract}

\section{INTRODUCTION}

The object of the present paper is a class of fullyactuated underwater vehicles whose thrust and torque controls can be assigned independently. While this configuration is typical for remotely operated vehicles (ROVs), it also exists in some autonomous underwater vehicles (AUVs).Underwater environment gives rise to several control difficulties. For instance, the dynamics of an underwater vehicle is highly nonlinear and characterized by a strong coupling between translational and rotational dynamics, mainly due to added mass effects [Fossen, 2002]. In addition, the key issue related to the control of AUVs is the lack of lightweight and reliable position sensors. A vision system, being lightweight, passive and adaptable, can be used, at least partially, to respond to this challenge. By using a camera as the primary sensor for relative position, the control problem can be cast into ImageBased Visual Servo (IBVS) control problem [Chaumette and Hutchinson, 2006], which opens the possibility to perform autonomous tasks in low-structured environments with no external assistance. Classical visual servo control was developed for serial-link robotic manipulators [Chaumette and Hutchinson, 2006] and more recently for aerial vehicles [Guenard et al., 2008], [Gonçalves et al., 2009]. In the underwater robotics field few attempts have been made to use vision sensors to perform tasks related to man-made structures such as pipeline following [Rives and Borrelly, 1997, Krupinski et al., 2012] using linear features, or station keeping [Lots et al., 2001] using point

* This work was supported by the French Centre National de la Recherche Scientifique (CNRS) within the PEPS CONGRE project. features. When the visual target is planar, an IBVS control scheme is proposed in [Benhimane and Malis, 2007] using the homography matrix that encodes transformation information between two images of the same planar target and that can be directly retrieved from the corresponding images. This homography-based visual servoing (HBVS) scheme is a purely kinematic control, initially designed for fully-actuated manipulators. However, its stability and convergence properties are only local and are not provable when the system's dynamics is taken into account. The HBVS problem has also been investigated for underactuated aerial vehicles [Metni et al., 2005], [de Plinval et al., 2013]. In [Metni et al., 2005], additional information such as the orientation measurement of the camera with respect to the target is assumed to be available. In contrast, the HBVS solution proposed in [de Plinval et al., 2013] only makes use of the homography matrix along with gyroscope's measurements. However, only local (exponential) stability is proved based on Lyapunov-like analysis. In fact, the consideration of the vehicle's dynamics within the control design is crucial to obtain provable (strong) stability. These works also show that significant efforts are required in order to eliminate the assumptions concerning the precise knowledge of environment geometry. The present study is an alignment with these efforts. Although the AUV system under consideration is fully-actuated, the strongly coupled translational and rotational dynamics represents another challenge. Moreover, in contrast with existing HBVS solutions, an important original outcome of the proposed control approach is related to the obtention of almost-global asymptotical stability by means of continuous feedback control. 
The paper is organized as follows. Notation, system modeling and HBVS problem are described in Section 2. Control design is presented in Section 3. In Subsection 3.1, an inner-loop control is proposed for the stabilisation of reference velocity setpoint, based on some modifications of the prior work [Krupinski et al., 2012]. In Subsection 3.2, we present the main result of the paper concerning the design of an outer-loop control directly based on the homography matrix, with the objective of assigning a reference velocities setpoint for the inner-loop control. Section 4 reports simulation results illustrating the performance of the control approach on a realistic AUV model. Finally, conclusions are given in Section 5.

\section{PRELIMINARY MATERIAL}

\subsection{Notation}

The following notation is used (see Fig. 1):

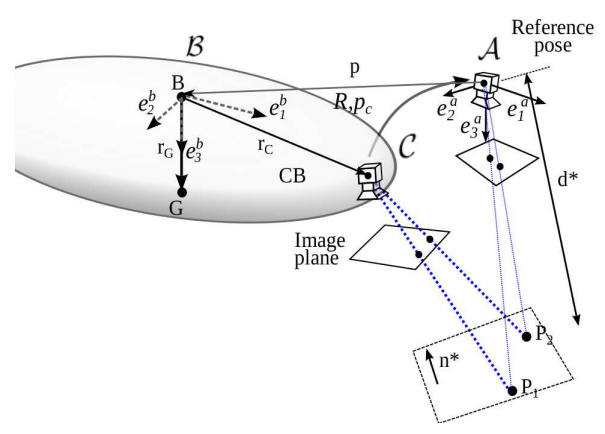

Fig. 1. Notation.

- $G$ and $B$ are the vehicle's center of mass and center of buoyancy, respectively, $m$ its mass and $\mathbf{J}_{0}$ its inertia matrix. Let $l$ denote the distance between $G$ and $B$.

- $\mathcal{A}=\left\{O ; \vec{e}_{1}^{a}, \vec{e}_{2}^{a}, \vec{e}_{3}^{a}\right\}$ is an inertial frame chosen such that its $\vec{e}_{3}^{a}$-axis points downwards and coincides with the gravity direction. $\mathcal{B}=\left\{B ; \vec{e}_{1}^{b}, \vec{e}_{2}^{b}, \vec{e}_{3}^{b}\right\}$ is a frame attached to the body whose origin coincides with the vehicle's center of buoyancy. $\mathcal{C}=\left\{C ; \vec{e}_{1}^{c}, \vec{e}_{2}^{c}, \vec{e}_{3}^{c}\right\}$ is a frame attached to the camera, which is displaced from the origin of $\mathcal{B}$ by a vector $\overrightarrow{B C}$ and keeps its base vectors parallel to those of $\mathcal{B}$. Let $\mathbf{r}_{C} \in \mathbb{R}^{3}$ and $\mathbf{r}_{G} \in \mathbb{R}^{3}$ denote the vectors of coordinates expressed in the frame $\mathcal{B}$ of $\overrightarrow{B C}$ and $\overrightarrow{B G}$, respectively.

- The orientation of the body-fixed frame $\mathcal{B}$ with respect to (w.r.t.) the inertial frame $\mathcal{A}$ is represented by the rotation matrix $\mathbf{R} \in \mathrm{SO}(3)$.

- The position vectors of the origins of the body-fixed frame $\mathcal{B}$ and the camera frame $\mathcal{C}$, expressed in the inertial frame $\mathcal{A}$, are denoted as $\mathbf{p}$ and $\mathbf{p}_{C}$, respectively. Their relation is $\mathbf{p}=\mathbf{p}_{C}-\mathbf{R} \mathbf{r}_{C}$.

- The angular velocity vector of the body-fixed frame $\mathcal{B}$ relative to the inertial frame $\mathcal{A}$, expressed in the frame $\mathcal{B}$, is denoted as $\boldsymbol{\Omega}=\left[\omega_{1}, \omega_{2}, \omega_{3}\right]^{\top} \in \mathbb{R}^{3}$. The translational velocity vectors of the origins and the frame $\mathcal{B}$ and the frame $\mathcal{C}$, expressed in the frame $\mathcal{B}$, are denoted as $\mathbf{V} \in \mathbb{R}^{3}$ and $\mathbf{V}_{C} \in \mathbb{R}^{3}$, respectively.

- $\left\{\mathbf{e}_{1}, \mathbf{e}_{2}, \mathbf{e}_{3}\right\}$ denotes the canonical basis of $\mathbb{R}^{3}$. $\mathbf{I}_{3}$ is the identity matrix of $\mathbb{R}^{3 \times 3}$. For all $\mathbf{u} \in \mathbb{R}^{3}$, the notation $\mathbf{u}_{\times}$ denotes the skew-symmetric matrix associated with the cross product by $\mathbf{u}$, i.e., $\mathbf{u}_{\times} \mathbf{v}=\mathbf{u} \times \mathbf{v}, \forall \mathbf{v} \in \mathbb{R}^{3}$. The
Euclidean norm in $\mathbb{R}^{n}$ is denoted as $|\cdot|$ and $(\cdot)^{\top}$ denotes the transpose operator.

- $\operatorname{sat}^{\delta}(\cdot) \in \mathbb{R}^{n}$, with $\delta>0$, is the classical saturation function defined as $\operatorname{sat}^{\delta}(\mathbf{x}) \triangleq \mathbf{x} \min (1, \delta /|\mathbf{x}|), \forall \mathbf{x} \in \mathbb{R}^{n}$.

- $\operatorname{satc}_{\boldsymbol{\Delta}}(\cdot) \in \mathbb{R}^{3}$, with some positive diagonal matrix $\boldsymbol{\Delta}=\operatorname{diag}\left(\left[\delta_{1}, \delta_{2}, \delta_{3}\right]\right)$, is a saturation function defined as $\operatorname{satc}_{\boldsymbol{\Delta}}(\mathbf{x}) \triangleq\left[\operatorname{sat}^{\delta_{1}}\left(x_{1}\right) \operatorname{sat}^{\delta_{2}}\left(x_{2}\right) \operatorname{sat}^{\delta_{3}}\left(x_{3}\right)\right]^{\top}, \forall \mathbf{x} \in \mathbb{R}^{3}$.

\subsection{System Modeling}

Without loss of generality, let us assume that $G$ lies on the $\vec{e}_{3}^{b}$-axis and under the center of buoyancy $B$ such that $\mathbf{r}_{G}=l \mathbf{e}_{3}$, i.e., bottom-heavy vehicle.

Define $\mathbf{W} \triangleq\left[\begin{array}{ll}\mathbf{V}^{\top} & \boldsymbol{\Omega}^{\top}\end{array}\right]^{\top} \in \mathbb{R}^{6}$. When characterized at the center of buoyancy $B$, the kinetic energy of the vehicle is given by (see [Leonard, 1997]):

$$
E_{B}=\frac{1}{2} \mathbf{W}^{\top} \mathbf{M}_{B} \mathbf{W}, \quad \text { with } \quad \mathbf{M}_{B} \triangleq\left[\begin{array}{cc}
m \mathbf{I}_{3} & -m \mathbf{r}_{G \times} \\
m \mathbf{r}_{G \times} & \mathbf{J}_{0}
\end{array}\right]
$$

According to Kirchhoff and Lamb theory [Lamb, 1932], the kinetic energy of the liquid surrounding the vehicle is given by:

$$
E_{F}=\frac{1}{2} \mathbf{W}^{\top} \mathbf{M}_{A} \mathbf{W}, \quad \text { with } \quad \mathbf{M}_{A} \triangleq\left[\begin{array}{ll}
\mathbf{M}_{A}^{11} & \mathbf{M}_{A}^{12} \\
\mathbf{M}_{A}^{21} & \mathbf{M}_{A}^{22}
\end{array}\right],
$$

where $\mathbf{M}_{A} \in \mathbb{R}^{6 \times 6}$ is known as the added mass matrix, which is constant and symmetric. The total kinetic energy of the body-fluid system is $E_{T}=E_{B}+E_{F}=\mathbf{W}^{\top} \mathbf{M}_{T} \mathbf{W}$, where the positive-definite matrix $\mathbf{M}_{T}$ is given by:

$$
\mathbf{M}_{T}=\mathbf{M}_{B}+\mathbf{M}_{A}=\left[\begin{array}{cc}
\mathbf{M} & \mathbf{D}^{\top} \\
\mathbf{D} & \mathbf{J}
\end{array}\right]
$$

with $\mathbf{M} \triangleq m \mathbf{I}_{3}+\mathbf{M}_{A}^{11}, \mathbf{J} \triangleq \mathbf{J}_{0}+\mathbf{M}_{A}^{22}, \mathbf{D} \triangleq m l \mathbf{e}_{3 \times}+\mathbf{M}_{A}^{21}$. The matrices $\mathbf{M}_{A}^{11}$ and $\mathbf{M}_{A}^{22}$ are often referred to as added mass and added inertia matrices, respectively. One derives the translational and rotational momentums as follows:

$$
\mathbf{P}=\frac{\partial E_{T}}{\partial \mathbf{V}}=\mathbf{M} \mathbf{V}-\mathbf{D} \boldsymbol{\Omega}, \quad \boldsymbol{\Pi}=\frac{\partial E_{T}}{\partial \boldsymbol{\Omega}}=\mathbf{J} \boldsymbol{\Omega}+\mathbf{D V}
$$

Then, the equations of motion satisfy [Leonard, 1997]:

$$
\left\{\begin{array}{l}
\dot{\mathbf{p}}=\mathbf{R V} \\
\dot{\mathbf{R}}=\mathbf{R} \boldsymbol{\Omega}_{\times} \\
\dot{\mathbf{P}}=\mathbf{P} \times \boldsymbol{\Omega}+\left(m g-F_{B}\right) \mathbf{R}^{\top} \mathbf{e}_{3}+\mathbf{F}_{D}+\mathbf{F}_{C} \\
\dot{\mathbf{\Pi}}=\mathbf{\Pi} \times \boldsymbol{\Omega}+\mathbf{P} \times \mathbf{V}+m g l \mathbf{e}_{3} \times \mathbf{R}^{\top} \mathbf{e}_{3}+\boldsymbol{\Gamma}_{D}+\boldsymbol{\Gamma}_{C}
\end{array}\right.
$$

The term $\left(m g-F_{B}\right) \mathbf{R}^{\top} \mathbf{e}_{3}$ is the contribution of both gravitational and buoyancy forces. The cross term $m g l \mathbf{e}_{3} \times$ $\mathbf{R}^{\top} \mathbf{e}_{3}$ represents the gravitational moment w.r.t. the center of buoyancy. The expansion of $\mathbf{P} \times \mathbf{V}$ in Eq. (1d) shows that the term $(\mathbf{M V}) \times \mathbf{V}$ should not be neglected in the control design due the added mass effects [Leonard, 1997]. The terms $\mathbf{F}_{D}$ and $\boldsymbol{\Gamma}_{D}$ represent the damping force and torque due to fluid pressure and viscous drag. Finally, $\mathbf{F}_{C} \in \mathbb{R}^{3}$ and $\boldsymbol{\Gamma}_{C} \in \mathbb{R}^{3}$ are the force and torque control vector inputs.

\subsection{Homography-based Visual Servo Control Problem}

The vehicle is assumed to be equipped with a camera, an Inertial Measurement Unit (IMU) and a Doppler velocity $\log$ (DVL). The IMU provides the measurements of the angular velocity $\boldsymbol{\Omega}$ and the gravitational direction $\mathbf{R}^{\top} \mathbf{e}_{3}$ (i.e., roll and pitch angles), whereas the DVL measures the translational velocity $\mathbf{V}$. 
A reference image of a planar target is taken at some desired pose (i.e., position and orientation). Based on this reference image and the current image, the control objective consists in stabilising the pose of the camera to the desired one. Assume that the camera provides the measurement of the homography matrix $\mathbf{H}$, which contains geometric information about the rotation and translation between two reference frames (see Fig. 1). The homography matrix $\mathbf{H}$ is given by [Benhimane and Malis, 2007]:

$$
\mathbf{H}=\mathbf{R}^{\top}-\left(1 / d^{*}\right) \mathbf{R}^{\top} \mathbf{p}_{C} \mathbf{n}^{* \top},
$$

where $d^{*}$ is the distance between the target plane and the camera optical center, and $\mathbf{n}^{*}=\left[\begin{array}{lll}n_{1}^{*} & n_{2}^{*} & n_{3}^{*}\end{array}\right]^{\top}$ is the unit vector normal to the target plane expressed in the reference camera frame.

Hypothesis 1. Assume that the reference image is taken when the $A U V$ stays in a horizontal plane. In addition, the inertial frame $\mathcal{A}$ are chosen attached to the reference pose of the camera (see Fig. 1).

Hypothesis 2. Assume that a rough knowledge of $\mathbf{n}^{*}$ is available such that one can choose a unit vector $\mathbf{m}^{*} \in \mathbb{S}^{2}$ such that $\mathbf{n}^{* \top} \mathbf{m}^{*}>0$.

The control objective can be stated as the stabilisation of $\mathbf{H}$ about the identity matrix $\mathbf{I}_{3}$, or equivalently the stabilisation of $\left(\mathbf{R}, \mathbf{p}_{C}\right)$ about $\left(\mathbf{I}_{3}, \mathbf{0}\right)$.

\section{CONTROL DESIGN}

The proposed control approach is split into two cascade parts. The first part -termed inner-loop control- ensures the stabilisation of the translational and angular velocities to a desired setpoint. The second one -termed outerloop control- is specifically designed from the homography matrix to define the desired velocities setpoint.

\subsection{Inner-loop Control}

The control objective taken consists in stabilising the AUV's velocities $(\mathbf{V}, \boldsymbol{\Omega})$ about the reference velocities $\left(\mathbf{V}_{r}, \boldsymbol{\Omega}_{r}\right)$, specified by the outer-loop control. The additional objective is the stabilisation of $\vec{e}_{3}^{b}$ about $\vec{e}_{3}^{a}$ or equivalently of $\mathbf{R e}_{3}$ about $\mathbf{e}_{3}$. This objective can be translated as the stabilisation of the AUV in a horizontal plane. To this purpose, let us define the reference angular velocity $\boldsymbol{\Omega}_{r}$ as:

$$
\mathbf{\Omega}_{r} \triangleq \omega_{3 r} \mathbf{e}_{3}+k_{\omega} \mathbf{e}_{3} \times \mathbf{R}^{\top} \mathbf{e}_{3},
$$

where $k_{\omega}$ is a positive gain and the third component $\omega_{3 r}$ of the reference angular velocity is dynamically assigned by the outer-loop control. The first two components of $\boldsymbol{\Omega}_{r}$ defined by the term $k_{\omega} \mathbf{e}_{3} \times \mathbf{R}^{\top} \mathbf{e}_{3}$ are dedicated to the stabilisation of $\mathbf{R e}_{3}$ about $\mathbf{e}_{3}$. The remaining degree of freedom $\omega_{3 r}$ can be independently used for other control objectives related to the yaw motion.

Define the velocity error variables

$$
\widetilde{\mathbf{V}} \triangleq \mathbf{V}-\mathbf{V}_{r}, \quad \widetilde{\boldsymbol{\Omega}} \triangleq \mathbf{\Omega}-\boldsymbol{\Omega}_{r} .
$$

Then, the control objective is equivalent to the stabilisation of $(\widetilde{\mathbf{V}}, \widetilde{\boldsymbol{\Omega}})$ about zero.

The inner-loop controller here proposed is reminiscent of the one in [Krupinski et al., 2012], but it is robustified by means of integral corrections. Let $\mathbf{z}_{V}$ and $\mathbf{z}_{\Omega}$ be the bounded conditional integrators of the linear and angular velocity errors, whose dynamics are given by:

$$
\begin{cases}\dot{\mathbf{z}}_{V}=-k_{z_{V}} \mathbf{z}_{V}+k_{z_{V}} \operatorname{sat}^{\delta_{V}}\left(\mathbf{z}_{V}+\widetilde{\mathbf{V}} / k_{z_{V}}\right), & \mathbf{z}_{V}(0)=\mathbf{0} \\ \dot{\mathbf{z}}_{\Omega}=-k_{z_{\Omega}} \mathbf{z}_{\Omega}+k_{z_{\Omega}} \operatorname{sat}^{\delta_{\Omega}}\left(\mathbf{z}_{\Omega}+\widetilde{\boldsymbol{\Omega}} / k_{z_{\Omega}}\right), & \mathbf{z}_{\Omega}(0)=\mathbf{0}\end{cases}
$$
with positive numbers $k_{z_{V}}, k_{z_{\Omega}}, \delta_{V}, \delta_{\Omega}$. Define the following augmented error variables:

$$
\overline{\mathbf{V}} \triangleq \widetilde{\mathbf{V}}+k_{i V} \mathbf{z}_{V}, \quad \overline{\mathbf{\Omega}} \triangleq \widetilde{\boldsymbol{\Omega}}+k_{i \Omega} \mathbf{z}_{\Omega},
$$

with positive integral gains $k_{i V}$ and $k_{i \Omega}$. Then, using Eqs. (1c), (1d), (4), (6), one obtains the following coupled dynamics of $\overline{\mathbf{V}}$ and $\overline{\boldsymbol{\Omega}}$ :

$$
\left\{\begin{aligned}
\mathbf{M} \dot{\overline{\mathbf{V}}}-\mathbf{D} \dot{\overline{\mathbf{\Omega}}} & =(\mathbf{M V}-\mathbf{D} \boldsymbol{\Omega})_{\times} \overline{\boldsymbol{\Omega}}+(\mathbf{M} \overline{\mathbf{V}}-\mathbf{D} \overline{\boldsymbol{\Omega}})_{\times} \boldsymbol{\Omega}_{r} \\
& +\left(m g-F_{B}\right) \mathbf{R}^{\top} \mathbf{e}_{3}+\mathbf{F}_{D}+\mathbf{F}+\mathbf{F}_{C} \\
\mathbf{J} \dot{\overline{\mathbf{\Omega}}}+\mathbf{D} \dot{\overline{\mathbf{V}}} & =(\mathbf{J} \boldsymbol{\Omega}+\mathbf{D V})_{\times} \overline{\mathbf{\Omega}}+(\mathbf{M V}-\mathbf{D} \boldsymbol{\Omega})_{\times} \overline{\mathbf{V}} \\
& +(\mathbf{J} \overline{\boldsymbol{\Omega}}+\mathbf{D} \overline{\mathbf{V}})_{\times} \boldsymbol{\Omega}_{r}+(\mathbf{M} \overline{\mathbf{V}}-\mathbf{D} \overline{\boldsymbol{\Omega}})_{\times} \mathbf{V}_{r} \\
& +m g l \mathbf{e}_{3} \times \mathbf{R}^{\top} \mathbf{e}_{3}+\boldsymbol{\Gamma}_{D}+\mathbf{\Gamma}+\boldsymbol{\Gamma}_{C}
\end{aligned}\right.
$$

with $\mathbf{F}$ and $\boldsymbol{\Gamma}$ computable by the controller and defined by

$$
\begin{aligned}
\mathbf{F} \triangleq & -\mathbf{M} \dot{\mathbf{V}}_{r}+\mathbf{D} \dot{\boldsymbol{\Omega}}_{r}+k_{i V} \mathbf{M} \dot{\mathbf{z}}_{V}-k_{i \Omega} \mathbf{D} \dot{\mathbf{z}}_{\Omega}+\left(\mathbf{M} \mathbf{V}_{r}-\mathbf{D} \boldsymbol{\Omega}_{r}\right)_{\times} \boldsymbol{\Omega}_{r} \\
& -k_{i \Omega}(\mathbf{M V}-\mathbf{D} \boldsymbol{\Omega})_{\times} \mathbf{z}_{\Omega}-\left(k_{i V} \mathbf{M} \mathbf{z}_{V}-k_{i \Omega} \mathbf{D} \mathbf{z}_{\Omega}\right)_{\times} \boldsymbol{\Omega}_{r} \\
\mathbf{\Gamma} \triangleq & -\mathbf{J} \dot{\boldsymbol{\Omega}}_{r}-\mathbf{D} \dot{\mathbf{V}}_{r}+k_{i \Omega} \mathbf{J} \dot{\mathbf{z}}_{\Omega}+k_{i V} \mathbf{D} \dot{\mathbf{z}}_{V} \\
& +\left(\mathbf{J} \boldsymbol{\Omega}_{r}+\mathbf{D} \mathbf{V}_{r}\right)_{\times} \boldsymbol{\Omega}_{r}+\left(\mathbf{M V} \mathbf{V}_{r}-\mathbf{D} \boldsymbol{\Omega}_{r}\right)_{\times} \mathbf{V}_{r} \\
& -k_{i \Omega}(\mathbf{J} \boldsymbol{\Omega}+\mathbf{D V})_{\times} \mathbf{z}_{\Omega}-k_{i V}(\mathbf{M V}-\mathbf{D} \boldsymbol{\Omega})_{\times} \mathbf{z}_{V} \\
& -\left(k_{i \Omega} \mathbf{J} \mathbf{z}_{\Omega}+k_{i V} \mathbf{D} \mathbf{z}_{V}\right)_{\times} \boldsymbol{\Omega}_{r}-\left(k_{i V} \mathbf{M} \mathbf{z}_{V}-k_{i \Omega} \mathbf{D} \mathbf{z}_{\Omega}\right)_{\times} \mathbf{V}_{r}
\end{aligned}
$$

From here, the paper's first result is proposed.

Proposition 3. Consider the error system (7a)-(7b) and apply the following controller:

$$
\left\{\begin{aligned}
\mathbf{F}_{C}= & -\operatorname{satc}_{\boldsymbol{\Delta}_{V}}\left(\mathbf{K}_{V} \overline{\mathbf{V}}\right)-(\mathbf{M} \overline{\mathbf{V}})_{\times} \boldsymbol{\Omega}_{r}+\mathbf{M}^{\top}\left(\overline{\boldsymbol{\Omega}}_{\times} \mathbf{V}_{r}\right) \\
& -\overline{\boldsymbol{\Omega}} \times\left(\mathbf{D} \boldsymbol{\Omega}_{r}\right)-\left(m g-F_{B}\right) \mathbf{R}^{\top} \mathbf{e}_{3}-\mathbf{F}_{D}-\mathbf{F} \\
\boldsymbol{\Gamma}_{C}= & -\operatorname{satc}_{\boldsymbol{\Delta}_{\Omega}}\left(\mathbf{K}_{\Omega} \overline{\boldsymbol{\Omega}}\right)-(\mathbf{I} \overline{\boldsymbol{\Omega}})_{\times} \boldsymbol{\Omega}_{r}+(\mathbf{D} \overline{\boldsymbol{\Omega}})_{\times} \mathbf{V}_{r}-\boldsymbol{\Gamma}_{D^{-}} \mathbf{\Gamma}
\end{aligned}\right.
$$

where $\mathbf{K}_{V}, \mathbf{K}_{\Omega}$ are some positive diagonal gain matrices and $\boldsymbol{\Delta}_{V}, \boldsymbol{\Delta}_{\Omega}$ are some positive diagonal matrices involved in the saturation functions. Assume that $\mathbf{V}_{r}, \boldsymbol{\Omega}_{r}$ and their derivative are bounded. Then,

(1) $\left(\mathbf{V}, \boldsymbol{\Omega}, \mathbf{R e}_{3}, \mathbf{z}_{V}, \mathbf{z}_{\Omega}\right)$ converges to $\left(\mathbf{V}_{r}, \boldsymbol{\Omega}_{r}, \pm \mathbf{e}_{3}, \mathbf{0}, \mathbf{0}\right)$ for all initial conditions.

(2) The equilibrium $\left(\mathbf{V}, \boldsymbol{\Omega}, \mathbf{R e}_{3}, \mathbf{z}_{V}, \mathbf{z}_{\Omega}\right)=\left(\mathbf{V}_{r}, \boldsymbol{\Omega}_{r}, \mathbf{e}_{3}, \mathbf{0}, \mathbf{0}\right)$ is almost globally asymptotically stable and locally exponentially stable. Conversely, the undesired equilibrium $\left(\mathbf{V}, \boldsymbol{\Omega}, \mathbf{R e}_{3}, \mathbf{z}_{V}, \mathbf{z}_{\Omega}\right)=\left(\mathbf{V}_{r}, \boldsymbol{\Omega}_{r},-\mathbf{e}_{3}, \mathbf{0}, \mathbf{0}\right)$ is unstable.

The proof is given in Appendix A. The knowledge about the derivative of the reference velocities $\mathbf{V}_{r}$ and $\boldsymbol{\Omega}_{r}$ are necessary to compute the feedback control $\mathbf{F}_{C}$ and $\boldsymbol{\Gamma}_{C}$. In the following, we show how this fact influences the HBVS outer-loop control design.

\subsection{Outer-loop Control: HBVS Control Design}

The outer-loop control design is based directly on the homography matrix $\mathbf{H}$. Control design difficulties lie in the fact that the depth $d^{*}$ and the normal vector $\mathbf{n}^{*}$ involved in the expression (2) of $\mathbf{H}$ are unknown and that this matrix only contains a coupled information of rotation and translation. For instance, let us recall a well-known result proposed in [Benhimane and Malis, 2007]. Let $\mathbf{e}_{p}, \mathbf{e}_{\Theta} \in \mathbb{R}^{3}$ denote the error vectors defined as:

$$
\mathbf{e}_{p} \triangleq\left(\mathbf{I}_{3}-\mathbf{H}\right) \mathbf{m}^{*}, \quad \mathbf{e}_{\Theta} \triangleq \operatorname{vex}\left(\mathbf{H}^{\top}-\mathbf{H}\right)
$$

with some arbitrary unit vector $\mathbf{m}^{*} \in \mathbb{S}^{2}$ satisfying Hypothesis 2. Then, the kinematic control law

$$
\mathbf{V}_{C}=-\lambda_{p} \mathbf{e}_{p}, \quad \boldsymbol{\Omega}=-\lambda_{\Theta} \mathbf{e}_{\Theta}
$$


with $\lambda_{p}, \lambda_{\Theta}$ some positive gains, makes the equilibrium $\left(\mathbf{R}, \mathbf{p}_{C}\right)=\left(\mathbf{I}_{3}, \mathbf{0}\right)$ locally asymptotically stable [Benhimane and Malis, 2007]. A weakness of this kinematic controller is the local basin of attraction of the equilibrium. On the other hand, due to the strongly coupled translational and rotational dynamics, this purely kinematic control approach may fail to guarantee the stability of the controlled system. In the present paper, we aim at working down to the dynamical level with the objective of yielding a controller with a significantly enlarged domain of stability and enhanced robustness.

3.2.1 Reference Translational Velocity Design: Differentiating $\mathbf{H}$ given by Eq. (2), one obtains

$$
\dot{\mathbf{H}}=-\boldsymbol{\Omega} \times \mathbf{H}-\left(1 / d^{*}\right) \mathbf{V}_{C} \mathbf{n}^{* \top} \text {. }
$$

It follows that the derivative of $\mathbf{e}_{p}$ defined in (9) satisfies $\dot{\mathbf{e}}_{p}=-\boldsymbol{\Omega} \times\left(\mathbf{e}_{p}-\mathbf{m}^{*}\right)+a^{*} \mathbf{V}_{C}, \quad a^{*} \triangleq\left(\mathbf{n}^{* \top} \mathbf{m}^{*}\right) / d^{*}$.

For control design insights, let us, for instance, consider the kinematic control design using the camera velocity $\mathbf{V}_{C}$ as control input, with the objective of stabilising $\mathbf{e}_{p}$ about zero globally. In view of Eq. (12), the control difficulty lies in the unknown multiplicative constant $a^{*}$. However, we know that it is positive in view of Hypothesis 2 .

Lemma 4. - Kinematic Control - Assume that $\boldsymbol{\Omega}$ is bounded for all time and consider the following auxiliary dynamics: $\quad \dot{\mathbf{z}}_{p}=-\boldsymbol{\Omega} \times \mathbf{e}_{p}, \quad \mathbf{z}_{p}(0)=\mathbf{0}$.

Then, the kinematic control law

$$
\mathbf{V}_{C}=\mathbf{V}_{C r} \triangleq-k_{1} \mathbf{e}_{p}-\mathbf{\Omega} \times \mathbf{z}_{p}
$$

globally asymptotically stabilise $\mathbf{e}_{p}$ about zero.

Proof: Using Eqs. (12), (13) and (14), one verifies that

$$
\dot{\mathbf{e}}_{p}=-\boldsymbol{\Omega} \times \mathbf{e}_{p}-a^{*} k_{1} \mathbf{e}_{p}-a^{*} \boldsymbol{\Omega} \times\left(\mathbf{z}_{p}-\mathbf{z}_{p}^{*}\right),
$$

with $\mathbf{z}_{p}^{*} \triangleq \mathbf{m}^{*} / a^{*}$. Consider the following Lyapunov function candidate $\mathcal{L}_{0} \triangleq 1 /\left(2 a^{*}\right)\left|\mathbf{e}_{p}\right|^{2}+1 / 2\left|\mathbf{z}_{p}-\mathbf{z}_{p}^{*}\right|^{2}$. Using Eqs. (13) and (15), one verifies that the derivative of $\mathcal{L}_{0}$ satisfies $\dot{\mathcal{L}}_{0}=-k_{1}\left|\mathbf{e}_{p}\right|^{2}$. From here, one ensures that $\mathcal{L}_{0}$ and, thus, $\mathbf{e}_{p}$ and $\mathbf{z}_{p}$ are bounded w.r.t. initial conditions. The resulting boundedness of $\dot{\mathbf{e}}_{p}$ given in (15) and, thus, of $\ddot{\mathcal{L}}_{0}$ implies the uniform continuity of $\dot{\mathcal{L}}_{0}$. Finally, the application of Barbalat's lemma (see [Khalil, 1992]) ensures the convergence of $\dot{\mathcal{L}}_{0}$ to zero. This concludes the proof.

In view of the relation $\mathbf{V}=\mathbf{V}_{C}-\boldsymbol{\Omega} \times \mathbf{r}_{C}$ and the kinematic control expression (14), one may define the reference velocity $\mathbf{V}_{r}$ as $\mathbf{V}_{r} \triangleq \mathbf{V}_{C r}-\boldsymbol{\Omega}_{r} \times \mathbf{r}_{C}$, with $\mathbf{V}_{C r} \triangleq-k_{1} \mathbf{e}_{p}-\boldsymbol{\Omega}_{r} \times \mathbf{z}_{p}$. As mentioned previously the derivative of $\mathbf{V}_{r}$ should be computable by the inner-loop control (8). However, since $\dot{\mathbf{e}}_{p}$ is not measurable, $\dot{\mathbf{V}}_{C r}$ and, thus, $\dot{\mathbf{V}}_{r}$ are not available to the computation of the innerloop control. The following modification to Lemma 4 is proposed.

Proposition 5. Let $k_{1}, k_{2}, k_{z}, \Delta, \nabla$ denote some positive constants. Consider the following augmented system:

$\left\{\begin{array}{l}\dot{\mathbf{z}}_{p}=-\boldsymbol{\Omega}_{r} \times \operatorname{sat}^{\nabla}\left(\mathbf{e}_{p}\right)-k_{z}\left(\mathbf{z}_{p}-\mathrm{sat}^{\Delta}\left(\mathbf{z}_{p}\right)\right), \mathbf{z}_{p}(0)=\mathbf{0} \\ \dot{\hat{\mathbf{e}}}_{p}=-\boldsymbol{\Omega} \times \hat{\mathbf{e}}_{p}-k_{2}\left(\hat{\mathbf{e}}_{p}-\mathbf{e}_{p}\right), \quad \hat{\mathbf{e}}_{p}(0)=\mathbf{e}_{p}(0)\end{array}\right.$

with $\Delta$ large enough such that $\Delta \geq 1 / a^{*}$. Consider the following reference translational velocity:

$$
\left\{\begin{array}{c}
\mathbf{V}_{r} \triangleq \mathbf{V}_{C r}-\boldsymbol{\Omega}_{r} \times \mathbf{r}_{C} \\
\mathbf{V}_{C r} \triangleq-k_{1} \hat{\mathbf{e}}_{p}-\boldsymbol{\Omega}_{r} \times \mathbf{z}_{p}
\end{array}\right.
$$

Choose the gains $k_{1}$ and $k_{2}$ such that

$$
k_{2}>4 a^{*} k_{1} \text {. }
$$

Assume that Hypothesis 2 is satisfied. Assume that the reference angular velocity $\boldsymbol{\Omega}_{r}$ and its derivative are bounded. Apply the inner-loop control (8) proposed in Proposition 3. Then, there exists a positive constant $\bar{\nabla}$ such that for all $\nabla>\bar{\nabla}, \mathbf{e}_{p}$ is globally stabilised about zero.

The proof is given in Appendix B. In view of Eq. (17), the definition of the reference translational velocity $\mathbf{V}_{r}$ depends on the reference angular velocity $\boldsymbol{\Omega}_{r}$. In the following, $\boldsymbol{\Omega}_{r}$ will be defined such that it and its derivative are bounded by some constants, as a necessary condition of Propositions 3 and 5 .

3.2.2 Reference Angular Velocity Design: As mentioned in inner-loop control design, only the third component $\omega_{3 r}$ of $\boldsymbol{\Omega}_{r}$ given in (3) is required to be defined by the outer-loop control. The derivative $\dot{\omega}_{3 r}$ is also needed for the computation of the inner-loop control.

Before proceeding the design for $\omega_{3 r}$, let us analyse the asymptotic behaviour of the homography matrix $\mathbf{H}$. Since $\mathbf{R e}_{3}$ (almost) globally converges to $\mathbf{e}_{3}$ as a result of Proposition 3, one ensures the convergence of $\mathbf{R}$ to $\mathbf{R}_{\psi}$ defined by:

$$
\mathbf{R}_{\psi} \triangleq\left[\begin{array}{ccc}
\cos \psi & -\sin \psi & 0 \\
\sin \psi & \cos \psi & 0 \\
0 & 0 & 1
\end{array}\right]
$$

For the sake of simplicity, assume that Hypothesis 2 is satisfied while choosing $\mathbf{m}^{*}=-\mathbf{e}_{3}$. This implies that $\mathbf{H e}_{3}-\mathbf{e}_{3}$ converges to zero as a result of Proposition 5. From here, one deduces

$$
\mathbf{H e}_{3}-\mathbf{e}_{3} \rightarrow\left(\mathbf{R}_{\psi}^{\top}-\mathbf{I}_{3}\right) \mathbf{e}_{3}+a^{*} \mathbf{R}_{\psi}^{\top} \mathbf{p}_{C} \rightarrow 0
$$

with $a^{*}=-n_{3}^{*} / d^{*}$. Therefore, $\mathbf{p}_{C}$ converges to $\frac{1}{a^{*}}\left(\mathbf{R}_{\psi}-\right.$ $\left.\mathbf{I}_{3}\right) \mathbf{e}_{3}$, which is null, and $\mathbf{H}$ converges to $\mathbf{R}_{\psi}^{\hat{a}^{*}}$. Additionally, From Eqs. (17), (B.1), and the convergence of $\left(\mathbf{e}_{p}, \hat{\mathbf{e}}_{p}, \dot{\mathbf{e}}_{p}, \widetilde{\mathbf{V}}, \widetilde{\mathbf{\Omega}}\right)$ to zero, one deduces that

$$
\mathbf{V}_{C} \rightarrow \mathbf{V}_{C r} \rightarrow-\left(1 / a^{*}\right) \omega_{3 r} \mathbf{e}_{3} \times \mathbf{m}^{*}=\mathbf{0}
$$

Denote $h_{i, j}$ as the component on the i-th line and $\mathrm{j}$-th column of $\mathbf{H}$. Now, the main result of the paper is stated, with proof given in Appendix C.

Theorem 6. Assume that Hypotheses 1 and 2 are satisfied with $\mathbf{m}^{*}=-\mathbf{e}_{3}$. Define the reference angular velocity $\boldsymbol{\Omega}_{\mathbf{r}} \triangleq \omega_{3 r} \mathbf{e}_{3}+k_{\omega} \mathbf{e}_{3} \times \mathbf{R}^{\top} \mathbf{e}_{3}$, where $\omega_{3 r}$ is the solution to the following system:

$$
\dot{\omega}_{3 r}=-k_{4} \omega_{3 r}-k_{3} \operatorname{sat}^{\Delta_{\omega}}\left(h_{1,2}\right), \quad \omega_{3 r}(0)=0,
$$

with $k_{3}, k_{4}$ some positive gains and $\Delta_{\omega}>1$. Define the reference translational velocity $\mathbf{V}_{r}$ as in Proposition 5 and apply the inner-loop control (8) given in Proposition 3. Then, the following properties hold:

(1) There exist only two isolated equilibria $\mathbf{H}=\mathbf{H}_{i}^{\star}$, $(i=1,2)$, with one stable and one unstable.

(2) The desired equilibrium $\mathbf{H}=\mathbf{I}_{3}$ is almost globally asymptotically stable and locally exponentially stable.

The existence of multiple equilibria of the closed-loop system is expected since there exists a topological obstruction to the existence of a globally asymptotically stable equilibrium to a continuous dynamical systems having rotational degrees of freedom (see [Bhat and Bernstein, 2000]). Consequently, the almost-global asymptotical stability is the best one can obtain with continuous feedback control, which is the result of the present paper. 


\section{SIMULATION RESULTS}

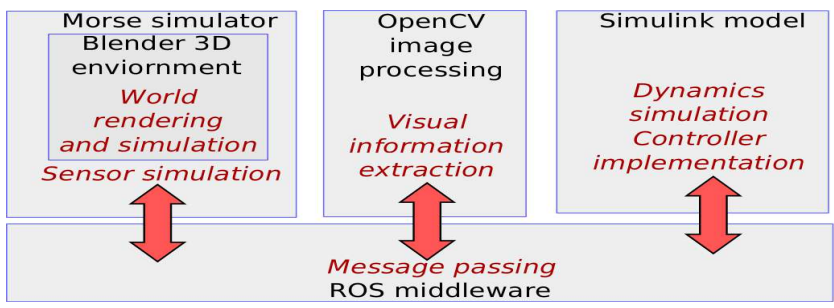

Fig. 2. Components of the AUV simulator

The proposed controller has been tested via a realistic simulation of a fully-actuated AUV model. A custom simulator has been developed (see Fig. 2). Equations of motion (1a)-(1d) and basic sensors are implemented in a Matlab/Simulink ${ }^{\circledR}$ model, while Morse simulator is used to generate the visual environment and the camera data (see Fig. 3). The images are processed to provide the homography matrix $\mathbf{H}$ at about $25 \mathrm{~Hz}$ by OpenCV functions integrated into a ROS communication graph.

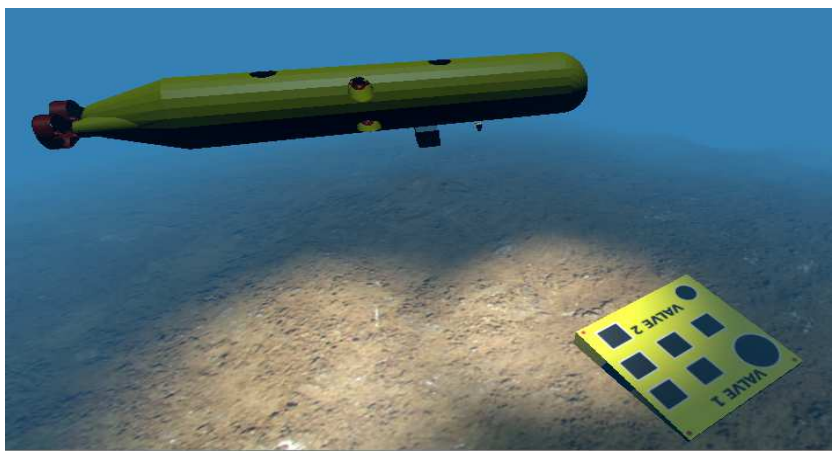

Fig. 3. Vehicle visualised in the simulated sea bottom environment above the visual target

The following model of hydrodynamic damping force and torque is used in the system dynamics [Fossen, 2002]:

$$
\left\{\begin{array}{l}
\mathbf{F}_{D}=-\mathbf{D}_{V l} \mathbf{V}_{a}-\mathbf{D}_{V n}\left[\left|V_{a 1}\right| V_{a 1}\left|V_{a 2}\right| V_{a 2}\left|V_{a 3}\right| V_{a 3}\right]^{\top} \\
\boldsymbol{\Gamma}_{D}=-\mathbf{D}_{\Omega l} \boldsymbol{\Omega}-\mathbf{D}_{\Omega n}\left[\left|\omega_{1}\right| \omega_{1}\left|\omega_{2}\right| \omega_{2}\left|\omega_{3}\right| \omega_{3}\right]^{\top}
\end{array}\right.
$$

with damping matrices $\mathbf{D}_{V l}, \mathbf{D}_{V n}, \mathbf{D}_{\Omega l}$ and $\mathbf{D}_{\Omega n}$, the apparent velocity $\mathbf{V}_{a}=\left[V_{a 1}, V_{a 2}, V_{a 3}\right]^{\top} \triangleq \mathbf{V}-\mathbf{R}^{\top} \mathbf{v}_{c u r}$, and the current velocity $\mathbf{v}_{\text {cur }}$ expressed in the inertial frame. The physical parameters of the simulated vehicle, given in Tab. 1, closely follow that of a real AUV.

\begin{tabular}{|c|c|}
\hline Specification & Numerical value \\
\hline Mass $m[k g]$ & 1000 \\
Volume $\left[\mathrm{m}^{3}\right]$ & 0.97 \\
$l[\mathrm{~m}]$ & 0.15 \\
$\mathbf{r}_{C}[\mathrm{~m}]$ & {$[1,0,0.5]$} \\
& {$\left[\begin{array}{cc}500-15 & -25 \\
-153000-10 \\
\mathbf{J}_{0}\left[\mathrm{~kg} \cdot \mathrm{m}^{2}\right] & -25-103000\end{array}\right]$} \\
& $10^{3} \operatorname{diag}(0.07,1.5,1.5)$ \\
$\mathbf{M}_{A}^{11}[\mathrm{~kg}]$ & $10^{3} \operatorname{diag}(1.5,15,15)$ \\
$\mathbf{M}_{A}^{22}\left[\mathrm{~kg} \cdot \mathrm{m}^{2}\right]$ & $\mathbf{0}^{3 \times 3}$ \\
$\mathbf{M}_{A}^{12}\left[\mathrm{~kg} \cdot \mathrm{m}^{2}\right]$ & $\operatorname{diag}(10,30,30)$ \\
$\mathbf{D}_{V l}\left[\mathrm{~kg} \cdot \mathrm{s}^{-1}\right]$ & $\operatorname{diag}(100,300,300)$ \\
$\mathbf{D}_{V n}\left[\mathrm{~kg} \cdot \mathrm{m}^{-1}\right]$ & $\operatorname{diag}(5,15,15)$ \\
$\mathbf{D}_{\Omega l}\left[\mathrm{~kg} \cdot \mathrm{m}^{2} \cdot \mathrm{s}^{-1}\right]$ & $\operatorname{diag}(50,150,150)$ \\
$\mathbf{D}_{\Omega n}[\mathrm{~N} \cdot \mathrm{m}]$ & \\
\hline
\end{tabular}

Table 1. Specifications of the simulated AUV.

- Simulation 1: This simulation is dedicated to the performance comparison between the proposed HBVS control approach and the "standard" kinematic HBVS (i.e., Eqs. (10)) in perfect situation where all measurements are perfect and the homography matrix $\mathbf{H}$ is directly calculated according to Eq. (2), with $d^{*}=3[\mathrm{~m}]$ and $\mathbf{n}^{*}=[-0.0858,0.1736,-0.9811]^{\top}=-\mathbf{R}_{10^{\circ}, 5^{\circ}, 0^{\circ}} \mathbf{e}_{3}$. In fact, for the two control approaches we apply the same inner-loop control (8), but the reference setpoints defined by the outer-loop control are differently specified by the two approaches. More precisely, for the inner-loop control we assume that the system's dynamics and parameters are perfectly known in the sense that "real" physical parameters of the vehicle and the expressions (21) of $\mathbf{F}_{D}$ and $\boldsymbol{\Gamma}_{D}$ are used in the computation of the inner-loop control. Thus, it is unnecessary to activate the integrator parts (i.e., setting $k_{i V}=k_{i \Omega}=0$ ). The gains and parameters (other than those involved in the integrators (5a)-(5b)) for the inner-loop control (8) are chosen as follows:

$$
\left\{\begin{array}{l}
\mathbf{K}_{V}=10^{3} \operatorname{diag}(0.93,0.464,2.5) \\
\mathbf{K}_{\Omega}=10^{4} \operatorname{diag}(0.759,5.623,1.8) \\
\boldsymbol{\Delta}_{V}=\operatorname{diag}(1500,1500,1500) \\
\boldsymbol{\Delta}_{\Omega}=\operatorname{diag}(375,750,750)
\end{array}\right.
$$

As for the outer-loop control corresponding to the proposed approach, the gains and parameters are given as:

- $k_{1}=1.5, k_{2}=4, k_{z}=8, \Delta=10, \nabla=5$;

- $k_{3}=0.81, k_{4}=1.8, \Delta_{\omega}=10, k_{\omega}=0.1$.

As for the outer-loop control corresponding to the "standard" kinematic HBVS, the reference velocities are set as:

$$
\left\{\begin{array}{l}
\boldsymbol{\Omega}_{r} \triangleq-\lambda_{\Theta} \mathbf{e}_{\Theta}, \quad \mathbf{V}_{C r} \triangleq-\lambda_{p} \mathbf{e}_{p} \\
\mathbf{V}_{r} \triangleq \mathbf{V}_{C r}-\Omega_{r} \times \mathbf{r}_{C}
\end{array}\right.
$$

where $\mathbf{e}_{p}$ and $\mathbf{e}_{\Theta}$ are given by Eq. (9) with $\mathbf{m}^{*}=-\mathbf{e}_{3}$. The gains are chosen as $\lambda_{p}=1.5$ and $\lambda_{\Theta}=0.9$, allowing the two control approaches to have closely similar convergence rates. The derivative terms of the reference velocities are set equal to zero, since the derivative terms $\dot{\mathbf{e}}_{p}$ and $\dot{\mathbf{e}}_{\Theta}$ are not available for the computation of the controller.

Initial conditions are as follows: $\mathbf{p}(0)=[-3,-2,-4]^{\top}[\mathrm{m}]$, $\mathbf{R}_{\phi, \theta, \psi}(0)=\mathbf{R}_{\frac{-\pi}{12}, \frac{\pi}{8}, \pi}, \mathbf{V}(0)=\boldsymbol{\Omega}(0)=\mathbf{0}[\mathrm{m} / \mathrm{s}]$. This implies that $\mathbf{p}_{C}(0) \approx[-4.1,-2.1,-3.9]^{\top}[m]$. The initial yaw error is very large, i.e., $\widetilde{\psi}(0)=\pi$, allowing one to verify the large stability domain of the proposed controller.

Variations w.r.t. time of the vehicle's position and orientation errors -corresponding to the standard kinematic HBVS and the proposed HBVS- are reported in Figs. 4 and 5, respectively. One observes that for both control approaches the position and orientation errors converge to zero despite large initial yaw error. However, the performance of the standard kinematic HBVS approach is rather poor. Too much oscillation occurs in position error and the vehicle even makes an unnecessary $2 \pi$-rotation about the roll axis. On the contrary, the performance of the proposed HBVS approach is quite satisfying (see Fig. 5).

- Simulation 2: This simulation is devised to test the robustness of the proposed controller. First, the homography matrix $\mathbf{H}$ is now estimated from the observed images using OpenCV functions. Second, to test the robustness w.r.t. imperfect knowledge on the external forces and torques, in the computation of the inner-loop control (8) we simply set to zero the terms $\mathbf{F}_{D}$ and $\boldsymbol{\Gamma}_{D}$, while their expression (21) is still used in the simulated dynamics. A sea current velocity $\mathbf{v}_{\text {cur }}=[0,1,0]^{\top}[\mathrm{m} / \mathrm{s}]$ is also introduced. Third, realistic actuation limitations are also taken into account by setting physical saturations for the control thrust force and torque vectors at $[ \pm 2000, \pm 2000, \pm 2000][N]$ and $[ \pm 500, \pm 1000, \pm 1000][N . m]$, respectively. Finally, the fol- 


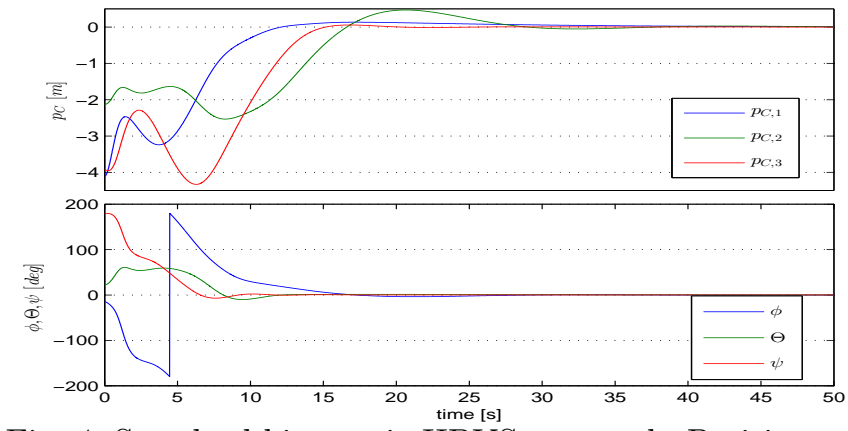

Fig. 4. Standard kinematic HBVS approach: Position and orientation errors vs. time (Sim. 1).

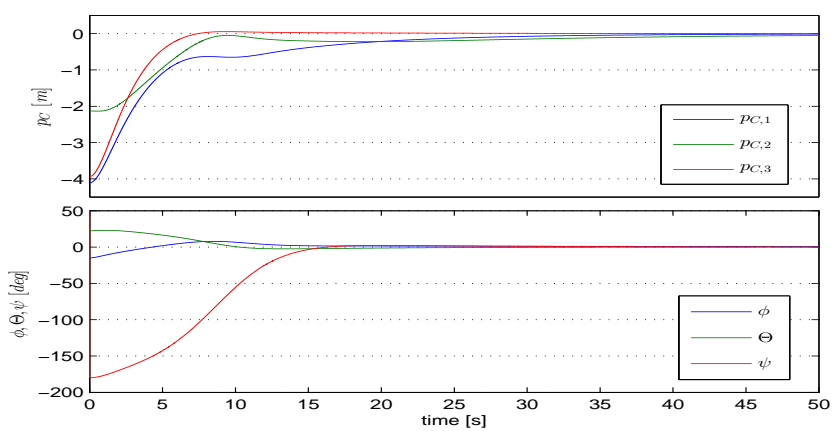

Fig. 5. Proposed HBVS approach: Position and orientation errors vs. time (Sim. 1).

lowing "erroneous" estimated parameters are used in the inner-loop control instead of the real values:

- $\hat{\mathbf{J}}_{0}=\operatorname{diag}(550,2600,3200)\left[\mathrm{kg} \cdot \mathrm{m}^{2}\right]$;

- $\hat{\mathbf{M}}_{A}^{11}=10^{3} \operatorname{diag}(0.05,1.33,1.33)[\mathrm{kg}]$;

- $\hat{\mathbf{M}}_{A}^{22}=10^{3} \operatorname{diag}(1.7,13,17)\left[\mathrm{kg} \cdot \mathrm{m}^{2}\right]$.

The control gains and other parameters involved in the computation of the control inputs are chosen as follows:

- $k_{i V}=0.2, k_{i \Omega}=0.05, k_{z V}=k_{z \Omega}=1, \delta_{V}=10, \delta_{\Omega}=10$;

- $\mathbf{K}_{V}, \mathbf{K}_{\Omega}, \boldsymbol{\Delta}_{V}$ and $\boldsymbol{\Delta}_{\Omega}$ are given in Eq. (22);

- $k_{1}=0.4, k_{2}=1, k_{z}=8, \Delta=10, \nabla=5$;

- $k_{3}=0.64, k_{4}=1.6, \Delta_{\omega}=10, k_{\omega}=0.1$.

The gains of the outer-loop control (i.e., $k_{1,2,3,4}$ ) are chosen smaller than in Sim. 1 so as to reduce the chattering effect due to measurement noises. Initial conditions of the vehicle are given by: $\mathbf{p}(0)=[0,-3,-4]^{\top}, \mathbf{R}_{\phi, \theta, \psi}(0)=\mathbf{R}_{\frac{\pi}{12}}, \frac{\pi}{8}, \frac{3 \pi}{4}$, $\mathbf{V}(0)=\boldsymbol{\Omega}(0)=\mathbf{0}$. Thus, $\mathbf{p}_{C}(0) \approx[-0.7,-2.1,-4.4]^{\top}[\mathrm{m}]$. Simulation results are reported in Figs. 6-9. Fig. 7 shows that the visual errors $\mathbf{e}_{p}$ and $h_{1,2}$ (computed from the estimated homography matrix) and the augmented variable $\hat{\mathbf{e}}_{p}$ converge to zero. One can observe the noise effects induced by imperfect homography matrix estimated from image processing. Fig. 6 shows the convergence of position and orientation errors to zero. Overshoots occur mainly due to important unknown disturbance force $\mathbf{F}_{D}$. However, thanks to the integral action in the inner-loop control, the controller manages to compensate for this force as well as other model errors, and thus still ensures asymptotical stability. The effect of the sea current on the integral terms $\mathbf{z}_{V}$ and $\mathbf{z}_{\Omega}$ is illustrated in Fig. 9, where the second component of $\mathbf{z}_{V}$ well converges to a nonnull value. The evolution of the control force and torque vectors w.r.t. time is shown in Fig. 8 where torque control saturation occurred in a short period of time marginally affects the overall control performance. The control forces in the directions $\vec{e}_{2}^{b}$ and $\vec{e}_{3}^{b}$ asymptotically converge to the values dictated by the compensation of the current and buoyancy, respectively. Finally, the reader is encouraged to watch a video clip showing this simulation result at http://youtu.be/07MhNBJJ-PA.

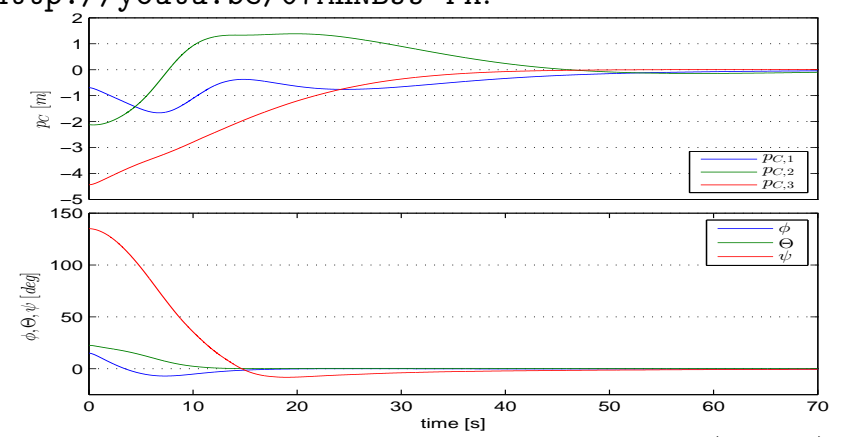

Fig. 6. Position and orientation errors vs. time (Sim. 2).

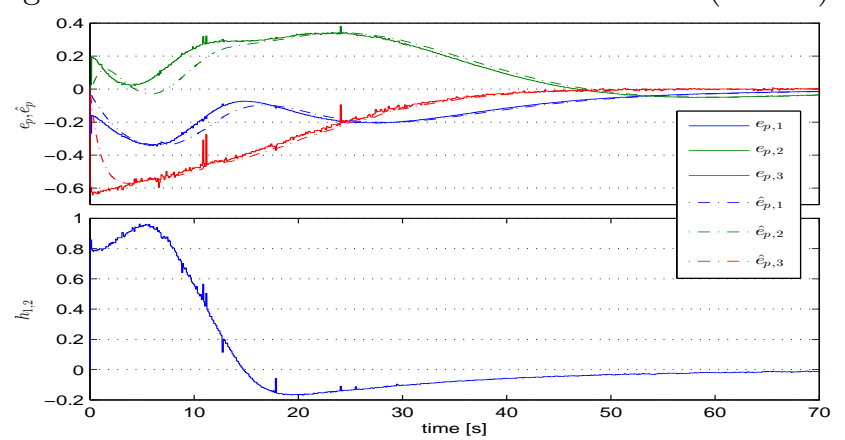

Fig. 7. Visual errors $\mathbf{e}_{p}, \hat{\mathbf{e}}_{p}$ and $h_{1,2}$ vs. time (Sim. 2).

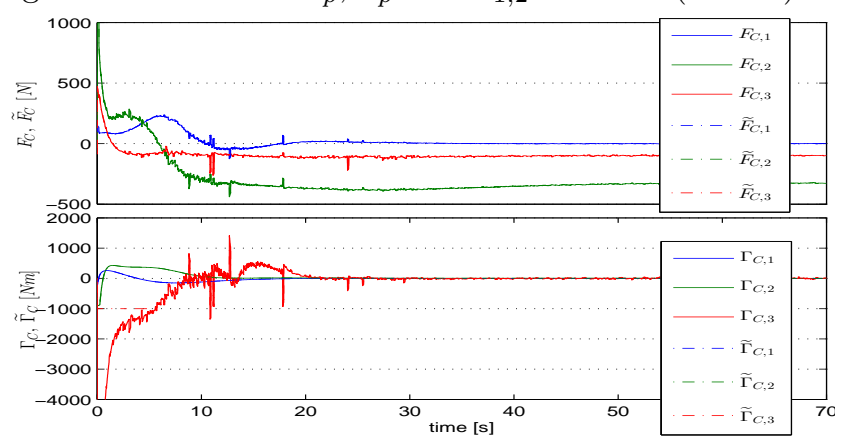

Fig. 8. $\mathbf{F}_{C}, \boldsymbol{\Gamma}_{C}$ calculated by the inner-loop control and the "saturated" values $\widetilde{\mathbf{F}}_{C}, \widetilde{\boldsymbol{\Gamma}}_{C}$ vs. time (Sim. 2).

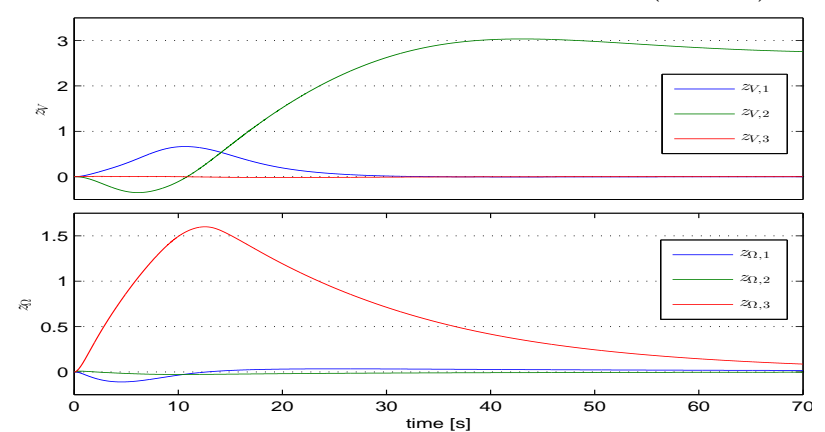

Fig. 9. $z_{V}$ and $z_{\Omega}$ vs. time (Sim. 2).

\section{CONCLUSIONS}

The stabilisation of fully-actuated AUVs using imagebased homography matrix opens a way to several new applications of autonomous vehicles, for example the inspection of underwater objects, too risky using traditional acoustic means. Underwater docking on flat targets can also benefit from this development. Using relatively available and cheap technology of underwater vision, navigation in a structured environment can be carried out using mathematically efficient formulation, rather than complicated 
pose reconstruction from multiple sensors. A testing campaign with a real AUV is now envisioned so as to validate the proposed approach in the challenging sea environment.

\section{REFERENCES}

S. Benhimane and E. Malis. Homography-based 2D Visual Tracking and Servoing. Int. J. of Robotics Research, 26 (7):661-676, 2007.

S.P. Bhat and D.S. Bernstein. A topological obstruction to continuous global stabilization of rotational motion and the unwinding phenomenon. Systems $\&$ Control Letters, 39(1):63-70, 2000.

F. Chaumette and S. Hutchinson. Visual servo control, Part I: Basic approaches. IEEE Robotics and Automation Magazine, 13(4):82-90, 2006.

H. de Plinval, P. Morin, P. Mouyon, and T. Hamel. Visual servoing for underactuated VTOL UAVs: A linear, Homography-Based framework. Int. J. of Robust and Nonlinear Control, 2013.

T. I. Fossen. Marine Control Systems. Marine Cybernetix AS, 2002.

T. F. Gonçalves, J. R. Azinheira, and P. Rives. Visionbased Autonomous Approach and Landing for an Aircraft using a Direct Visual Tracking Method. In Int. Conf. on Informatics in Control, Automation and Robotics, pages 94-101, 2009.

N. Guenard, T. Hamel, and R. Mahony. A practical visual servo control for an unmanned aerial vehicle. IEEE Trans. on Robotics, 24(2):331-340, 2008.

M.-D. Hua, T. Hamel, P. Morin, and C. Samson. A control approach for thrust-propelled underactuated vehicles and its application to VTOL drones. IEEE Trans. on Automatic Control, 54(8):1837-1853, 2009.

H. K. Khalil. Nonlinear systems. Macmillan Publishing Company, 1992.

S. Krupinski, G. Allibert, M.-D. Hua, and T. Hamel. Pipeline tracking for fully-actuated autonomous underwater vehicle using visual servo control. In American Control Conf., pages 6196-6202, 2012.

H. Lamb. Hydrodynamics. Cambridge Univ. Press, 1932.

N. E. Leonard. Stability of a bottom-heavy underwater vehicle. Automatica, 33(3):331-246, 1997.

J.-E Lots, D. M. Lane, E. Trucco, and F. Chaumette. A 2D visual servoing for underwater vehicle station keeping. In IEEE Int. Conf. on Robotics and Automation, pages 2767-2772, 2001.

N. Metni, T. Hamel, and F. Derkx. Visual Tracking Control of Aerial Robotic Systems with Adaptive Depth Estimation. In IEEE Conf. on Decision and Control, pages 6078-6084, 2005.

P. Rives and J.-J. Borrelly. Underwater Pipe Inspection Task using Visual Servoing Techniques. In IEEE Int. Conf. on Intelligent Robots and Syst., pages 63-68, 1997.

Appendix A. PROOF OF PROPOSITION 3 The proof is based on the analysis of the Lyapunov function candidate $\mathcal{L}_{\text {inner }} \triangleq \frac{1}{2} \overline{\mathbf{W}}^{\top} \mathbf{M}_{T} \mathbf{\mathbf { W }}+m g l\left(1-\mathbf{e}_{3}^{\top} \mathbf{R}^{\top} \mathbf{e}_{3}\right)$, with $\mathbf{\mathbf { W }} \triangleq\left[\overline{\mathbf{V}}^{\top} \overline{\boldsymbol{\Omega}}^{\top}\right]^{\top}$. After some tedious computation, it can be verified that the derivative of $\mathcal{L}_{v}$ along any solution to the closed-loop system satisfies

$$
\begin{aligned}
\dot{\mathcal{L}}_{\text {inner }}= & -\overline{\mathbf{V}}^{\top} \operatorname{satc}_{\boldsymbol{\Delta}_{V}}\left(\mathbf{K}_{V} \overline{\mathbf{V}}\right)-\overline{\mathbf{\Omega}}^{\top} \operatorname{satc}_{\boldsymbol{\Delta}_{\Omega}}\left(\mathbf{K}_{\Omega} \overline{\mathbf{\Omega}}\right) \\
& -m g l k_{\omega}\left|\mathbf{e}_{3} \times \mathbf{R}^{\top} \mathbf{e}_{3}\right|^{2} .
\end{aligned}
$$

By application of Barbalat's lemma, one deduces the convergence of $\dot{\mathcal{L}}_{\text {inner }}$ and, thus, of $\overline{\mathbf{V}}, \overline{\mathbf{\Omega}}$ and $1-\mathbf{e}_{3}^{\top} \mathbf{R}^{\top} \mathbf{e}_{3}$ to zero. The latter implies that $\mathbf{R e}_{3}$ converges to $\pm \mathbf{e}_{3}$. Let us now prove the local exponential stability (L.E.S.) of the equilibrium $\left(\overline{\mathbf{V}}, \overline{\mathbf{\Omega}}, \mathbf{R e}_{3}\right)=\left(\mathbf{0}, \mathbf{0}, \mathbf{e}_{3}\right)$. By denoting $\Theta$ the angle between $\mathbf{R e}_{3}$ and $\mathbf{e}_{3}$, i.e., $\cos (\Theta)=\mathbf{e}_{3}^{\top} \mathbf{R} \mathbf{e}_{3}$, around a small neighborhood of the equilibrium $\left(\overline{\mathbf{V}}, \overline{\mathbf{\Omega}}, \mathbf{R e}_{3}\right)=$ $\left(\mathbf{0}, \mathbf{0}, \mathbf{e}_{3}\right)$ the Lyapunov function $\mathcal{L}_{\text {inner }}$ can be locally approximated by $\mathcal{L}_{\text {inner }} \approx 0.5 \overline{\mathbf{W}}^{\top} \mathbf{M}_{T} \overline{\mathbf{W}}+0.5 \mathrm{mgl} \Theta^{2}$ and its derivative (A.1) is approximatively given by $\dot{\mathcal{L}}_{\text {inner }} \approx$ $-\overline{\mathbf{V}}^{\top} \mathbf{K}_{V} \overline{\mathbf{V}}-\overline{\mathbf{\Omega}}^{\top} \mathbf{K}_{\Omega} \overline{\boldsymbol{\Omega}}-m g l k_{\omega} \Theta^{2}$. As a consequence, there exists a positive number $\alpha$ such that locally $\dot{\mathcal{L}}_{\text {inner }} \leq$ $-\alpha \mathcal{L}_{\text {inner }}$. This ensures the L.E.S. of $\mathcal{L}_{\text {inner }}$ about zero and, thus, the L.E.S. of the equilibrium $\left(\overline{\mathbf{V}}, \overline{\mathbf{\Omega}}, \mathbf{R e}_{3}\right)=$ $\left(\mathbf{0}, \mathbf{0}, \mathbf{e}_{3}\right)$. Finally, the exponential convergence of $\overline{\mathbf{V}}$ and $\overline{\boldsymbol{\Omega}}$ to zero naturally leads to same property of $\widetilde{\mathbf{V}}$ and $\widetilde{\boldsymbol{\Omega}}$ as a consequence of the technical lemma 7 (Append. D).

The proof of instability of the equilibrium $\left(\overline{\mathbf{V}}, \overline{\mathbf{\Omega}}, \mathbf{R e}_{3}\right)=$ $\left(\mathbf{0}, \mathbf{0},-\mathbf{e}_{3}\right)$ is based on the Chetaev's theorem. Define $\mathbf{y}=\left[\begin{array}{lll}y_{1} & y_{2} & y_{3}\end{array}\right]^{\top} \triangleq \mathbf{e}_{3}+\mathbf{R e}_{3}$ and consider the following continuously differentiable function: $\mathcal{S}(\mathbf{y}) \triangleq y_{3}=1+$ $\mathbf{e}_{3}^{\top} \mathbf{R e}_{3} \geq 0$, which is null at the origin, i.e., $\mathcal{S}(\mathbf{0})=0$. For some positive number $r>0$, define a set $U_{r} \triangleq\{\mathbf{y} \mid$ $\mathcal{S}(\mathbf{y})>0,|\mathbf{y}|<r\}$, and note that $U_{r}$ is non-null for all $r>0$. By neglecting all high-order terms, the derivative of $\mathcal{S}$ can be approximatively given by

$\dot{\mathcal{S}} \approx \mathbf{e}_{3}^{\top} \mathbf{R} \boldsymbol{\Omega}_{r \times} \mathbf{e}_{3}=k_{\omega}\left|\mathbf{e}_{3} \times \mathbf{R e}_{3}\right|^{2}=k_{\omega}\left|\mathbf{e}_{3} \times \mathbf{y}\right|^{2}=k_{\omega}\left(y_{1}^{2}+y_{2}^{2}\right)$. The positivity of $y_{3}$ is equivalent to the positivity of $y_{1}^{2}+y_{2}^{2}$. Thus, for all $\mathbf{y} \in U_{r}$ one ensures that $\dot{\mathcal{S}}>0$. Since all the conditions of Chetaev's theorem are satisfied [Khalil, 1992], the origin $\mathbf{y}=0$ of the linearized system is unstable.

\section{Appendix B. PROOF OF PROPOSITION 5}

Using Eqs. (12) and (17), one deduces

$$
\dot{\mathbf{e}}_{p}=-\boldsymbol{\Omega}_{\times} \mathbf{e}_{p}-a^{*} \boldsymbol{\Omega}_{r \times} \overline{\mathbf{z}}_{p}-a^{*} k_{1} \hat{\mathbf{e}}_{p}+\gamma(\widetilde{\mathbf{V}}, \widetilde{\boldsymbol{\Omega}}),
$$
with $\gamma(\widetilde{\mathbf{V}}, \widetilde{\boldsymbol{\Omega}}) \triangleq a^{*}\left(\widetilde{\mathbf{V}}+\widetilde{\boldsymbol{\Omega}}_{\times} \mathbf{r}_{c}\right)+\widetilde{\boldsymbol{\Omega}}_{\times} \mathbf{m}^{*}, \overline{\mathbf{z}}_{p} \triangleq \mathbf{z}_{p}-\mathbf{z}_{p}^{*}$. From here, the proof proceeds by three steps:

Step 1: We will show that $\mathbf{z}_{p}$ is bounded by some constant. Consider the positive function $\mathcal{S}_{1} \triangleq 0.5\left|\mathbf{z}_{p}\right|^{2}$. Its derivative satisfies (using (16)) $\dot{\mathcal{S}}_{1} \leq-k_{z}\left|\mathbf{z}_{p}\right|^{2}+\left|\mathbf{z}_{p}\right|\left(\bar{\Omega}_{r} \nabla+k_{z} \Delta\right)$, with $\bar{\Omega}_{r} \triangleq \sup \left(\left|\boldsymbol{\Omega}_{r}\right|\right)$. From here, it is straightforward to deduce that $\forall t \geq 0$

$\left|\mathbf{z}_{p}(t)\right| \leq\left(\bar{\Omega}_{r} \nabla\right) / k_{z}+\Delta=\Delta\left(1+\alpha \bar{\Omega}_{r}\right)$, with $\alpha \triangleq \nabla /\left(k_{z} \Delta\right)$.

Step 2: We will show next that there exists a time instant $T$ such that $\forall \tau \geq T$ one has $\left|\mathbf{e}_{p}(\tau)\right| \leq \nabla$ and, thus, $\operatorname{sat}^{\nabla}\left(\mathbf{e}_{p}(\tau)\right)=\mathbf{e}_{p}(\tau)$. Denote $\mathbf{X} \triangleq\left[\begin{array}{ll}\mathbf{x} & \mathbf{y}\end{array}\right]^{\top} \in \mathbb{R}^{6}$, with $\mathbf{x} \triangleq \mathbf{R} \hat{\mathbf{e}}_{p}, \mathbf{y} \triangleq \mathbf{R e}_{p}$. One verifies from (16) and (B.1) that

$$
\dot{\mathbf{X}}=\underbrace{\left[\begin{array}{cc}
-k_{2} \mathbf{I}_{3} & k_{2} \mathbf{I}_{3} \\
-a^{*} k_{1} \mathbf{I}_{3} & \mathbf{0}
\end{array}\right]}_{=: \mathbf{A} \in \mathbb{R}^{6 \times 6}} \mathbf{X}+\underbrace{\left[\mathbf{R}\left(-a^{*} \boldsymbol{\Omega}_{r \times} \overline{\mathbf{z}}_{p}+\gamma(\widetilde{\mathbf{V}}, \widetilde{\boldsymbol{\Omega}})\right)\right]}_{=: \mathbf{B} \in \mathbb{R}^{6}}
$$

The condition (18) ensures that $\mathbf{A}$ has two triple distinct real negative eigenvalues $\lambda_{1,2}\left(\lambda_{1}<\lambda_{2}<0\right)$ given by $\lambda_{1,2}=0.5\left(-k_{2} \mp \sqrt{k_{2}^{2}-4 a^{*} k_{1} k_{2}}\right)$. This implies that $\mathbf{A}$ is diagonalisable and can be decomposed in the Jordan normal form $\mathbf{A}=\mathbf{P} \boldsymbol{\Lambda} \mathbf{P}^{-1}$, with $\boldsymbol{\Lambda}=\operatorname{diag}\left(\lambda_{1} \mathbf{I}_{3}, \lambda_{2} \mathbf{I}_{3}\right)$ and

$$
\mathbf{P}=\left[\begin{array}{cc}
\frac{\lambda_{1} \mathbf{I}_{3}}{\eta_{1}} & \frac{\lambda_{2} \mathbf{I}_{3}}{\eta_{2}} \\
\frac{-a_{1}^{*} k_{1} \mathbf{I}_{3}}{\eta_{1}} & \frac{-a_{1}^{*} k_{1} \mathbf{I}_{3}}{\eta_{2}}
\end{array}\right], \mathbf{P}^{-1}=\frac{1}{\lambda_{2}-\lambda_{1}}\left[\begin{array}{cc}
-\eta_{1} \mathbf{I}_{3} & \frac{-\lambda_{2} \eta_{1} \mathbf{I}_{3}}{a_{1}^{*} k_{1}} \\
\eta_{2} \mathbf{I}_{3} & \frac{\lambda_{1} \eta_{2} \mathbf{I}_{3}}{a_{1}^{*} k_{1}}
\end{array}\right]
$$

By simple calculations, it can be verified that 


$$
\begin{aligned}
& \left(\lambda_{2}-\lambda_{1}\right)^{2}\left|\mathbf{P}^{-1} \mathbf{X}\right|^{2}=\eta_{1}^{2}\left|\mathbf{x}+\frac{\lambda_{2}}{\left(a^{*} k_{1}\right)} \mathbf{y}\right|^{2}+\eta_{2}^{2}\left|\mathbf{x}+\frac{\lambda_{1} \mathbf{y}}{a^{*} k_{1}}\right|^{2} \\
& =\left|\sqrt{\eta_{1}^{2}+\eta_{2}^{2}}\left(\mathbf{x}+\frac{\lambda_{1} \mathbf{y}}{a^{*} k_{1}}\right)+\frac{\eta_{1}^{2}\left(\lambda_{2}-\lambda_{1}\right) \mathbf{y}}{a^{*} k_{1} \sqrt{\eta_{1}^{2}+\eta_{2}^{2}}}\right|^{2}+\frac{\eta_{1}^{2} \eta_{2}^{2}\left(\lambda_{2}-\lambda_{1}\right)^{2}|\mathbf{y}|^{2}}{\left(a^{*} k_{1}\right)^{2}\left(\eta_{1}^{2}+\eta_{2}^{2}\right)},
\end{aligned}
$$

which allows one to deduce

$$
\left|\mathbf{P}^{-1} \mathbf{X}\right| \geq \frac{\eta_{1} \eta_{2}}{a^{*} k_{1} \sqrt{\eta_{1}^{2}+\eta_{2}^{2}}}|\mathbf{y}|=\frac{\eta_{1} \eta_{2}}{a^{*} k_{1} \sqrt{\eta_{1}^{2}+\eta_{2}^{2}}}\left|\mathbf{e}_{p}\right|
$$

On the other hand, as a result of Proposition 3, $\widetilde{\Omega}$ and $\widetilde{\mathbf{V}}$ are uniformly continuous and bounded, and converge asymptotically to zero. Consequently, $\gamma(\widetilde{\mathbf{V}}, \widetilde{\boldsymbol{\Omega}})$ also converges to zero. Thus, for some positive number $\varepsilon$ (to be specified hereafter) there exists a time instant $T_{1}$ such that $\forall t \geq T_{1}$ one has $|\gamma(\widetilde{\mathbf{V}}, \widetilde{\boldsymbol{\Omega}})| \leq \varepsilon$. Then, $\forall t \geq T_{1}$ one verifies

$$
\begin{gathered}
\left|\mathbf{P}^{-1} \mathbf{B}\right|=\frac{\sqrt{\lambda_{1}^{2} \eta_{2}^{2}+\lambda_{2}^{2} \eta_{1}^{2}}}{a^{*} k_{1}\left(\lambda_{2}-\lambda_{1}\right)}\left|-a^{*} \boldsymbol{\Omega}_{r \times} \overline{\mathbf{z}}_{p}+\gamma(\widetilde{\mathbf{V}}, \widetilde{\boldsymbol{\Omega}})\right| \\
\quad \leq \frac{\sqrt{\lambda_{1}^{2} \eta_{2}^{2}+\lambda_{2}^{2} \eta_{1}^{2}}}{a^{*} k_{1}\left(\lambda_{2}-\lambda_{1}\right)}\left(\left(a^{*} \Delta\left(1+\alpha \bar{\Omega}_{r}\right)+1\right) \bar{\Omega}_{r}+\varepsilon\right) .
\end{gathered}
$$

Using Eq. (B.2), the derivative of $\mathcal{S}_{2} \triangleq 0.5\left|\mathbf{P}^{-1} \mathbf{X}\right|^{2}$ satisfies

$$
\begin{aligned}
\mathcal{S}_{2} & =\left(\mathbf{P}^{-1} \mathbf{X}\right)^{\top} \boldsymbol{\Lambda}\left(\mathbf{P}^{-1} \mathbf{X}\right)+\left(\mathbf{P}^{-1} \mathbf{X}\right)^{\top}\left(\mathbf{P}^{-1} \mathbf{B}\right) \\
& \leq \lambda_{2}\left|\mathbf{P}^{-1} \mathbf{X}\right|^{2}+\left|\mathbf{P}^{-1} \mathbf{X}\right|\left|\mathbf{P}^{-1} \mathbf{B}\right| .
\end{aligned}
$$

Since $\mathbf{z}_{p}, \boldsymbol{\Omega}_{r}, \widetilde{\boldsymbol{\Omega}}$ and $\widetilde{\mathbf{V}}$ are bounded, $\mathbf{B}$ and $\mathbf{P}^{-1} \mathbf{B}$ are also bounded. From Eq. (B.5) and the definition of $\mathcal{S}_{2}$, one ensures that $\mathbf{P}^{-1} \mathbf{X}$ and $\mathbf{X}$ are bounded w.r.t. initial conditions. Consequently, $\mathbf{e}_{p}$ and $\hat{\mathbf{e}}_{p}$ remain bounded w.r.t. initial conditions. Then, it is straightforward to verify that $\dot{\mathbf{e}}_{p}, \dot{\hat{\mathbf{e}}}_{p}$ and $\dot{\mathbf{z}}_{p}$ are bounded w.r.t. initial conditions, which implies the uniform continuity of $\mathbf{e}_{p}, \hat{\mathbf{e}}_{p}$ and $\mathbf{z}_{p}$.

Since $\mathbf{X}$ remains bounded w.r.t. initial conditions on the time-interval $\left[0, T_{1}\right]$ (as proved previously), from Eq. (B.5) and the definition of $\mathcal{S}_{2}$ there exists another time-instant $T>T_{1}$ such that $\forall \tau \geq T$ one has

$$
\left|\mathbf{P}^{-1} \mathbf{X}(\tau)\right| \leq\left(-\lambda_{2}\right)^{-1} \sup _{t \geq T}\left(\left|\mathbf{P}^{-1} \mathbf{B}(t)\right|\right) .
$$

In view of Eqs. (B.3), (B.4) and (B.6) one deduces that

$$
\left|\mathbf{e}_{p}(\tau)\right| \leq \bar{\nabla}+\varepsilon \frac{\sqrt{\left(\eta_{1}^{2}+\eta_{2}^{2}\right)\left(\lambda_{1}^{2} \eta_{2}^{2}+\lambda_{2}^{2} \eta_{1}^{2}\right)}}{\lambda_{2}\left(\lambda_{1}-\lambda_{2}\right) \eta_{1} \eta_{2}}, \forall \tau \geq T,
$$

with

$$
\bar{\nabla} \triangleq \frac{\sqrt{\left(\eta_{1}^{2}+\eta_{2}^{2}\right)\left(\lambda_{1}^{2} \eta_{2}^{2}+\lambda_{2}^{2} \eta_{1}^{2}\right)}}{\lambda_{2}\left(\lambda_{1}-\lambda_{2}\right) \eta_{1} \eta_{2}}\left(a^{*} \Delta\left(1+\alpha \bar{\Omega}_{r}\right)+1\right) \bar{\Omega}_{r}>0 .
$$

Therefore, if $\nabla$ is chosen larger than $\bar{\nabla}$ (i.e., $\nabla>\bar{\nabla}$ ) and if $\varepsilon$ is chosen such that

$$
0<\varepsilon<\frac{(\nabla-\bar{\nabla}) \lambda_{2}\left(\lambda_{1}-\lambda_{2}\right) \eta_{1} \eta_{2}}{\sqrt{\left(\eta_{1}^{2}+\eta_{2}^{2}\right)\left(\lambda_{1}^{2} \eta_{2}^{2}+\lambda_{2}^{2} \eta_{1}^{2}\right)}}
$$

then one deduces from inequality (B.7) that $\left|\mathbf{e}_{p}(\tau)\right|<\nabla$, $\forall \tau \geq T$, and thus $\operatorname{sat}^{\nabla}\left(\mathbf{e}_{p}(\tau)\right)=\mathbf{e}_{p}(\tau)$.

Step 3: Consider the Lyapunov candidate function

$$
\mathcal{L} \triangleq 1 /\left(2 a^{*}\right)\left|\mathbf{e}_{p}\right|^{2}+k_{1} /\left(2 k_{2}\right)\left|\hat{\mathbf{e}}_{p}\right|^{2}+1 / 2\left|\overline{\mathbf{z}}_{p}\right|^{2} .
$$

Using the following property [Hua et al., 2009]:

$$
\left|\operatorname{sat}^{\Delta}(\mathbf{x}+\mathbf{c})-\mathbf{c}\right| \leq|\mathbf{x}|, \forall(\mathbf{c}, \mathbf{x}) \in \mathbb{R}^{3} \times \mathbb{R}^{3} \text { with }|\mathbf{c}| \leq \Delta,
$$

one deduces

$$
\begin{aligned}
\dot{\mathcal{L}}= & -k_{1}\left|\hat{\mathbf{e}}_{p}\right|^{2}+\overline{\mathbf{z}}_{p}^{\top} \boldsymbol{\Omega}_{r \times}\left(\mathbf{e}_{p}-\operatorname{sat}^{\nabla}\left(\mathbf{e}_{p}\right)\right)+1 / a^{*} \mathbf{e}_{p}^{\top} \gamma(\widetilde{\mathbf{V}}, \widetilde{\mathbf{\Omega}}) \\
& -k_{z} \overline{\mathbf{z}}_{p}^{\top}\left(\overline{\mathbf{z}}_{p}+\mathbf{z}_{p}^{*}-\operatorname{sat}^{\Delta}\left(\overline{\mathbf{z}}_{p}+\mathbf{z}_{p}^{*}\right)\right) \\
\leq & -k_{1}\left|\hat{\mathbf{e}}_{p}\right|^{2}+\overline{\mathbf{z}}_{p}^{\top} \boldsymbol{\Omega}_{r \times}\left(\mathbf{e}_{p}-\operatorname{sat}^{\nabla}\left(\mathbf{e}_{p}\right)\right)+1 / a^{*} \mathbf{e}_{p}^{\top} \gamma(\widetilde{\mathbf{V}}, \widetilde{\boldsymbol{\Omega}}) .
\end{aligned}
$$

Since $\operatorname{sat}^{\nabla}\left(\mathbf{e}_{p}(\tau)\right)=\mathbf{e}_{p}(\tau)$ and $\left|\mathbf{e}_{p}(\tau)\right| \leq \nabla, \forall \tau \geq T$ (as proved in Step 2), one obtains

$$
\dot{\mathcal{L}}(\tau) \leq-k_{1}\left|\hat{\mathbf{e}}_{p}(\tau)\right|^{2}+\nabla / a^{*}|\gamma(\widetilde{\mathbf{V}}(\tau), \widetilde{\boldsymbol{\Omega}}(\tau))| .
$$
As proved previously, $\mathbf{e}_{p}, \hat{\mathbf{e}}_{p}$ and $\mathbf{z}_{p}$ cannot escape in finitetime. Thus, $\mathcal{L}(t)$ remains bounded on the time-interval $[0, T]$. In addition, $\mathbf{e}_{p}(\tau), \hat{\mathbf{e}}_{p}(\tau), \mathbf{z}_{p}(\tau)$ and, thus, $\mathcal{L}(\tau)$, $\forall \tau \geq T$, remain bounded by some positive constants independent from all initial conditions (as proved previously). Since the equilibrium $\left(\widetilde{\mathbf{V}}, \widetilde{\mathbf{\Omega}}, \mathbf{R}^{\top} \mathbf{e}_{3}\right)=\left(\mathbf{0}, \mathbf{0}, \mathbf{e}_{3}\right)$ is locally exponentially stable as a result of Proposition 3 , there exist some time-instant $T_{2}>T$ and some positive constants $\alpha_{1}$ and $\alpha_{2}$ such that

$$
|\gamma(\widetilde{\mathbf{V}}(\tau), \widetilde{\boldsymbol{\Omega}}(\tau))| \leq \alpha_{1} e^{-\alpha_{2} \tau}, \quad \forall \tau \geq T_{2} .
$$

From Eqs. (B.8) and (B.9), one deduces

$$
\dot{\mathcal{L}}(\tau) \leq-k_{1}\left|\hat{\mathbf{e}}_{p}(\tau)\right|^{2}+\left(\alpha_{1} \nabla\right) / a^{*} e^{-\alpha_{2} \tau}, \quad \forall \tau \geq T_{2} .
$$

Consequently, by integration one deduces

$$
\int_{T_{2}}^{\infty}\left|\hat{\mathbf{e}}_{p}(\tau)\right|^{2} d \tau \leq \frac{\alpha_{1} \nabla e^{-\alpha_{2} T_{2}}}{k_{1} \alpha_{2} a^{*}}+\frac{1}{k_{1}}\left(\mathcal{L}\left(T_{2}\right)-\mathcal{L}(\infty)\right) .
$$

From here, the resulting boundedness of integral term $\int_{T_{2}}^{\infty}\left|\hat{\mathbf{e}}_{p}(\tau)\right|^{2} d \tau$ and the uniform continuity of $\hat{\mathbf{e}}_{p}$ implies the convergence of $\hat{\mathbf{e}}_{p}$ to zero (Barbalat's lemma).

From Eq. (16), one verifies that $\dot{\hat{\mathbf{e}}}_{p}$ can be rewritten as $\dot{\hat{\mathbf{e}}}_{p}(t)=\mathbf{a}(t)+\mathbf{b}(t)$, with $\mathbf{a}(t) \triangleq k_{2} \mathbf{e}_{p}$ the uniformly continuous term and $\mathbf{b}(t) \triangleq-\boldsymbol{\Omega}_{\times} \hat{\mathbf{e}}_{p}-k_{2} \hat{\mathbf{e}}_{p}$ the vanishing term. Then, the application of the extended Barbalat's lemma [Hua et al., 2009] ensures the convergence of $\dot{\hat{\mathbf{e}}}_{p}$ to zero, which in turn implies the convergence of $\mathbf{e}_{p}$ to zero.

\section{Appendix C. PROOF OF THEOREM 6}

From (20), it is straightforward that $\omega_{3 r}$ and $\dot{\omega}_{3 r}$ remain bounded by $k_{3} \Delta_{\omega} / k_{4}$ and $2 k_{3} \Delta_{\omega}$, respectively. The boundedness of $\omega_{3 r}$ and $\dot{\omega}_{3 r}$ is a necessary condition of Propositions 3 and 5 . One ensures that at the zero dynamics $\dot{\psi}=\omega_{3}=\omega_{3 r}$ and $h_{1,2}=\sin \psi \leq 1<\Delta_{\omega}$. Thus, at the zero dynamics, the dynamics of $\omega_{3 r}$ given by (20) satisfies

$$
\dot{\omega}_{3 r}=-k_{4} \omega_{3 r}-k_{3} \sin \psi \text {. }
$$

Consider the positive function $\mathcal{S}_{\Theta} \triangleq k_{3}(1-\cos \psi)+\frac{1}{2} \omega_{3 r}^{2}$. Using (C.1), one obtains $\dot{\mathcal{S}}_{\Theta}=-k_{4} \omega_{3 r}^{2} \leq 0$. The application of LaSalle principle ensures the convergence of $\dot{\mathcal{S}}_{\Theta}$ and, thus, of $\omega_{3 r}$ to zero. Then, Barbalat's lemma ensures the convergence of $\dot{\omega}_{3 r}$ to zero. One deduces the convergence of $\sin \psi$ to zero. From here, one deduces the existence of two isolated equilibria of $\mathbf{H}$ corresponding to two values of $\psi=\psi_{1}^{\star} \triangleq 0$ and $\psi=\psi_{2}^{\star} \triangleq \pi$. The equilibrium $\psi=0$ corresponds to the desired equilibrium $\mathbf{H}=\mathbf{H}_{1}^{\star} \triangleq \mathbf{I}_{3}$ and the other one $\psi=\pi$ corresponds to the undesired equilibrium $\mathbf{H}=\mathbf{H}_{2}^{\star} \triangleq \operatorname{diag}(-1,-1,1)$.

To prove the local stability results, it suffices to study the linearized system about the equilibrium. For the equilibrium $\psi=0$, the linearized system is

$$
\tilde{\psi}=\omega_{3}, \quad \dot{\omega}_{3}=-k_{4} \omega_{3}-k_{3} \tilde{\psi}
$$

with $\tilde{\psi}=\psi$. Since its characteristic polynomial $p^{2}+k_{4} p+$ $k_{3}$ is Hurwitz, the equilibrium $\psi=\psi_{1}^{\star}=0$ is exponentially stable. On the other hand, the linearized systems for the equilibrium $\psi \equiv \pi$ is given by:

$$
\overline{\tilde{\psi}}=\omega_{3}, \quad \dot{\omega}_{3}=-k_{4} \omega_{3}+k_{3} \tilde{\psi}
$$

with $\tilde{\psi}=\psi-\pi$. Using Hurwitz criteria, one easily verifies that the origin of this linearized system is unstable.

\section{Appendix D. TECHNICAL LEMMA}

Lemma 7. (Omitted proof) If $\mathbf{x}(t)+k \int_{t_{0}}^{t} \mathbf{x}(\tau) d \tau$, with $\mathbf{x} \in \mathbf{R}^{n}$ and $k>0$, converges exponentially to some constant vector, then $\mathbf{x}(t)$ converges exponentially to zero. 